\title{
Fuzzy Knowledge Representation and Reasoning of the Smart Grid Based on Medium Logic and Its Application
}

\author{
Wang Jian, Wang Qiang, Ma WeiQing \\ The Department of information technology \\ Shanxi Electric Power Corporation \\ Taiyuan,China \\ tywangjian@vip.sina.com
}

\begin{abstract}
Medium logic completely reflects both the contrary relation and the contradictory relation. This paper introduces fuzzy privative and opposition privative in traditional knowledge representation. It takes a power transmission and distribution program for example, adopts a ratio function of distance to define the fuzzy set's member functions. Moreover, one confirmed method is presented to define the $\lambda$ value as well as the threshold $\tau$ value in fuzzy production rules. Finally it discusses fuzzy knowledge reasoning by a specific example.
\end{abstract}

Keywords-fuzzy knowledge representation; medium logic; truth value; smart grid; fuzzy production rules

\section{INTRODUCTION}

Grid is related to the lifeline of the national economy, basic industries and public utilities. Currently, the smart grid is becoming the research topic of many countries and regions, will also become the only way to develop the modern power industry. Smart grid, also known as Intelli-grid, is a transmission and distribution system combines information, digital and other cutting-edge technology; which development goal is to build the energy-efficient, environmentally friendly, efficient, reliable, stable, modern grid.

Today, with the increasing demand for electricity and the expanding scale of the power gird, smart grid fuzzy knowledge representation and reasoning method is particularly important. In the study of knowledge, the distinction of knowledge representation and processing is the foundation of the knowledge based processing. Some researchers have proposed medium logic research ideas and methods on the issue of representation and processing of uncertain knowledge and its negative. The medium logic system not only distinguish clear knowledge and fuzzy knowledge, and also distinguish the fuzzy privative relations hip and opposition privative relationship of knowledge. This theory has important implications for the construction of Chinese smart grid information platform.

Based on medium logic system, this paper explore fuzzy knowledge and its negative representation and reasoning by a specific example of power transmission and distribution.

\author{
Yao Dahai \\ Smart Grid Dep \\ Huada Tianyuan (Beijing) Electric Porwer Tecnaology \\ Co., LTD \\ Beijing, China \\ acprimer.yao@gmail.com
}

\section{THE MEDIUM LOGIC SYSTEM OVERVIEW}

The medium logic(ML) system was initiated in 1985 by Zhu Wu-Jia and Xiao Xi-ArL. Medium logic system is a fully formal the natural reasoning system, its syntax and semantic theory reflects complete negation relationship between knowledge. Medium predicate logic MF is a subsystem of the ML.

In the medium predicate logic, set $\mathrm{P}$ for a monadic predicate (concept or property), The opposition of $\mathrm{P}$ is called $\neg P$, then $\mathrm{P}$ and $\neg P$ represent a pair of opposite relationship with the predicate. We unconditional admit: There exists a predicate $\mathrm{P}$, and object $\mathrm{x}$, such that $P(x)$ and $\neg P(x)$ are partially true, such $x$ called intermediary object of $P(x)$ and $\neg P(x)$. Having such properties is referred to as $\sim P$, we call $\sim P$ the medium predicate of $P$ and $\neg P(x)$ (or fuzzy negative predicate of $\mathrm{P}$ ). So $\mathrm{P}$ and $\sim P$ represent a negative relationship with fuzzy predicate.

Definition 1 Medium predicate logic MF's $\lambda$ assignment $R_{\lambda}(\lambda \in(0,1))$, is designated according to the following rules and by individual domain and each constant symbol, function symbol, predicate symbols:

For each constant symbol,we designate an element in D associated with them.

For each n-ary function symbol, we designate a mapping function from $D^{n}$ to $D$ associated with them.

For each $n$-ary predicate symbol, we designate a mapping function from $D^{n}$ to $[0,1]$ associated with them, as well as:

$A$ is a atomic formula, $\mathrm{R}_{\lambda}(A)$ is assigned in $[0,1]$;

$$
R_{\lambda}(A)+R_{\lambda}(\neg A)=1 \text {; }
$$

$\mathrm{R}_{\lambda}(A \rightarrow B)=\operatorname{Max}\left(1-\mathrm{R}_{\lambda}(A), \mathrm{R}_{\lambda}(B)\right)$

\section{THE FUZZY KNOWLEDGE REPRESENTATION OVERVIEW}

The fuzzy facts in real life can be represented with the fuzzy predicates in the medium logic system. On the basis of traditional logical conjunction $\neg, \wedge, \vee, \rightarrow, \leftrightarrow$, we introduce fuzzy negation $\sim$, implication symbol $\neg$ and true value degree symbol \{, to expand the traditional logical expression,And combine them to a more complex facts and concepts. Among them, traditional logic $\neg$ can be defined by $\sim$ and $\neg: \neg \mathrm{A}=\mathrm{A} \rightarrow \sim \mathrm{A}$ or $\neg \mathrm{A}=\neg \mathrm{A} \vee \sim \mathrm{A}$. 
For fuzzy predicate expression, according to the specific knowledge area, each fuzzy predicate expression $\mathrm{P}(\mathrm{x})$ is assigned a true value in the interval $[0,1]$.

Definition 2 We introduce the symbol " +", for the fuzzy predicate $\mathrm{A}, \sim+\mathrm{A}$ represents a close to A part in $\sim \mathrm{A}$. We introduce the symbol “ -”, for the fuzzy predicate A, -A represents a close to $\neg A$ part in $\sim A$.

\section{THE APPLICATION OF THE MEDIUM LOGIC FUZZY} KNOWLEDGE REPRESENTATION REASONING IN SMART GRID

Grid has the large scale model complex multistage multilevel, and other notable features 。 Especially with the solar wind power hydraulic power and other renewable energy gradually access network and distributed energy technology unceasing development, the scale of power grid will be more big complexity higher wider distribution. The following analysis of the smart grid information platform application needs, with medium logic fuzzy knowledge representation systems theory knowledge classification timeshare conducive to reasonable classification storage grid data for further analysis and decision-making.

To reasonable electrical transmission and distribution is a particularly important part of a series of links in the smart grid. An important feature of the smart grid in that the adaptive, self-adjustment function,following we take a power transmission and distribution program for example,representing by medium logic combined with the fuzzy theory.

The auxiliary decision role is to help monitor monitoring the current smart grid operation normal degree of hierarchies according to the usual actual operation , convenient managers timely find problems and take further measures. Set the amount of electricity of the region is $m$, actual consumption is $n$, and power balance is $t=m-n$, then the monitoring level division depends on $t, n$ according to the following principles:

If $t$ is small, whatever the actual output $m$ and actual consumption are, we recognize it as A level.

If $\mathrm{t}$ is quite small and $\mathrm{n}$ is low, we recognize it as good, so it is normal to regard it as B level.

If $\mathrm{t}$ is quite big and $\mathrm{n}$ is high, it is the critical degree, and we regard it as $\mathrm{C}$ level, which means manual supervision is required.

If $t$ is pretty big, we regard it as warning level, this means artificial debugging and maintenance treatment is strongly recommended.

\section{A. Medium logic representation}

In order to make the decision support automation,we use the medium predicate calculus proposition to represent the guidelines. In order to facilitate the distinction between predicate symbols and function symbols in the logical predicate calculus symbols. We use capital letters to represent predicate symbols, and lowercase letters to represent function symbols. Set electricity in a day for domain, For any $X$ belonging to the domain, we introduce the necessary function and predicate symbols. According to definition 2 :
(1) $\sim^{+} \operatorname{MUCH}(t(X))$ : indicates power balance of $\mathrm{X}$ is relatively big;

(2) $\sim^{-} \operatorname{MUCH}(t(X))$ : indicates power balance of $\mathrm{X}$ is relatively small;

(3) $\neg M U C H(t(X))$ : indicates power balance of $\mathrm{X}$ is quite small;

(4) $\sim^{+} \operatorname{MUCH}(n(X))$ : indicates output of electricity of $\mathrm{X}$ is relatively big;

Applying logic expression into different hierarchies, Rules (1) can be expressed as: $\neg \operatorname{MUCH}(t(X)) \rightarrow \mathrm{A}$.

Likewise, rules (2) can be expressed as: $\sim^{-} \operatorname{MUCH}(t(X)) \wedge\left[\sim^{-} \operatorname{MUCH}(n(X)) \vee \neg \operatorname{MUCH}(n(X))\right] \rightarrow \mathrm{B}$.

Rules (3): $\sim^{-} \operatorname{MUCH}(t(X)) \wedge \sim^{+} \operatorname{MUCH}(n(X)) \rightarrow C$.

Rules (4): $\sim^{+} \operatorname{MUCH}(t(X)) \rightarrow D$.

\section{B. Truth degree}

Definition 4 Set $X$ be a non-empty set, for any a,b $\in X$ there exists only one real number $d(a, b)$ for corresponding, and meet the requirements

(1) $d(a, b)=d(b, a)$;

$(2) d(a, b) \geqslant 0, d(a, b)=O$ if and only if $a=b$;

(3) $d(a, b)+d(b, c) \geqslant d(a, c)$;

Then $\mathrm{d}$ is the distance of $\mathrm{X}$, this paper use the Euclidean distance in the case of one-dimensional, scilicet $d(a, b)=|a-b|$.

Definition 5 Given a non-empty domain of individuals D, $\mathrm{f}$ is a n-ary Numerical value mapping of $\mathrm{D}$. For any $\mathrm{x} \in \mathrm{D}$, subset $T \subset R^{n}$ and $F \subset R^{n}$ satisfied: if $f(x) \in T \Leftrightarrow P(x)$ and $f(x) \in F \Leftrightarrow \neg P(x)$, then $\mathrm{T}$ and $\mathrm{F}$ is respectively called "truth value area" and "false value area" of predicate $P$.

It's easy to know: the "truth value area" of predicate P is also the "false value area" of predicate $\neg P$.

Definition 6 if $\mathrm{f}$ is a one-ary numerical value mapping, scilicet $f: D \rightarrow R$, The true value of the corresponding numerical area of the predicate $\mathrm{P}$ is $\left[\alpha_{F}-\varepsilon_{F}, \alpha_{T}+\varepsilon_{T}\right]$, we call $\alpha$ is standard degree of $\mathrm{P}$.

We can use figure 1 to illustrate the relationship between predicate in one-ary numerical mapping and numerical area.

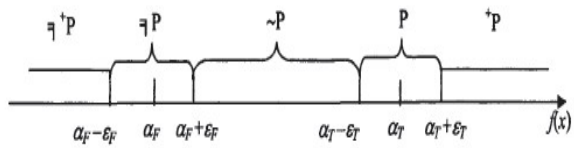

Figure 1 relationship between predicate in one-ary numerical mapping and numerical area

We call, $\alpha_{T}$ is the standard degree of P, $\alpha_{F}$ is the standard degree of $\neg P$. If $\alpha_{T}>\alpha_{F}$, then predicate $\mathrm{P}$ is called positive predicate; on the contrary we call $\mathrm{P}$ negative predicate.

for individual $\mathrm{x}$, we choose the corresponding numerical area length in $\sim p$ as a reference to decide the accurate level of predicate $\mathrm{p}(\mathrm{x})$ 's value. And then, we give the truth degree function about positive predicate $P$.

Definition 7 Distance ratio function relative to $\mathrm{P} h_{T}: f(D) \rightarrow R$,if $y=f(x) \in f(D)$, then 


$$
\mathrm{h}_{T}(x)= \begin{cases}0 & x<=\alpha_{F}+\varepsilon_{F} \\ \frac{d\left(x, \alpha_{F}+\varepsilon_{F}\right)}{d\left(\alpha_{F}+\varepsilon_{F}, \alpha_{T}-\varepsilon_{T}\right)} & \alpha_{F}+\varepsilon_{F}<x<\alpha_{T}-\varepsilon_{T} \\ 1 & x>=\alpha_{T}-\varepsilon_{T}\end{cases}
$$

Obviously, predicate “ $M U C H$ ” is fuzzy predicate. Due to the imbalance of power generation electricity consumption levels, zoning can be based on geography and generation capacity. The assumptions is divided into a, b, c, d four regions,we organize the capacity of calendar year(with 2007 2011 5 years' data as an example, balance power(different between output and consumption) in 4 regions, the results are as followed in Table 1:

Table 1 City power distribution statistics results 1 (power unit: *10 $\left.10^{5} \mathrm{KW} . \mathrm{h}\right)$

\begin{tabular}{|c|c|c|c|c|c|}
\hline year & region & $\begin{array}{c}\text { High power } \\
\text { generation(a } \\
\text { bove) }\end{array}$ & $\begin{array}{c}\text { Low power } \\
\text { generation } \\
\text { (below) }\end{array}$ & $\begin{array}{c}\text { High power } \\
\text { balance } \\
\text { (above) }\end{array}$ & $\begin{array}{c}\text { Low pow } \\
\text { balance } \\
\text { (below) }\end{array}$ \\
\hline 2007 & a & 1150 & 730 & 320 & 10 \\
\hline 2007 & b & 1210 & 810 & 500 & 60 \\
\hline 2007 & c & 1370 & 1000 & 610 & 50 \\
\hline 2007 & d & 1470 & 980 & 440 & 30 \\
\hline 2008 & a & 1260 & 740 & 400 & 70 \\
\hline 2008 & b & 1170 & 850 & 370 & 10 \\
\hline 2008 & c & 1380 & 1050 & 490 & 40 \\
\hline 2008 & d & 1510 & 1020 & 330 & 60 \\
\hline 2009 & a & 1280 & 720 & 360 & 30 \\
\hline 2009 & b & 1320 & 820 & 410 & 20 \\
\hline 2009 & c & 1490 & 1110 & 420 & 50 \\
\hline 2009 & d & 1590 & 1000 & 540 & 60 \\
\hline 2010 & a & 1310 & 770 & 510 & 30 \\
\hline 2010 & b & 1440 & 910 & 660 & 10 \\
\hline 2010 & c & 1520 & 1140 & 280 & 20 \\
\hline 2010 & d & 1630 & 1070 & 270 & 50 \\
\hline 2011 & a & 1410 & 830 & 290 & 40 \\
\hline 2011 & b & 1470 & 910 & 390 & 50 \\
\hline 2011 & c & 1600 & 1150 & 550 & 60 \\
\hline 2011 & d & 1630 & 1080 & 430 & 40 \\
\hline
\end{tabular}

To comprehensive analysis survey data in the same area over the years,we can average them respectively, then get the data in table 2. According to how much the survey year, An elastic region are determined for each class of data, the elastic region is determined by a given elastic amplitude $\mathcal{E}$. We believe that: the more the year of survey is, the more accurate the comprehensive data, the smaller the elastic region. In this example, we may wish to take the elastic range of high power generation $\varepsilon_{T}=70$, take the elastic range of low power generation $\varepsilon_{F}=20$.

When we discuss the degree of the true value of specific data $X$ for fuzzy predicate $M U C H$ or $\neg M U C H$,we can adopt distance concept. This is very natural for the true value the higher the degree, the farther away from the data region.

As can be seen from the data of Table 2, high power of the region $d$ is the highest. High power generation in region $\mathrm{D}$ is certainly also a high power generation in other regions.
Low power of the region a is the lowest, low pow generation is region a is certainly also a low power generation in other regions. The same is true for the power balance. Combined with the characteristic of the data, we adopt one dimensional Euclidean distance,denoted $d(x, y)$, scilicet $d(x, y)=|x-y|$.

Table 2 City power distribution statistics results 2 (power unit: *105KW.h)

\begin{tabular}{|c|c|c|cc|}
\hline & & & & \\
\hline $\mathrm{a}$ & 1282 & 758 & 376 & 36 \\
\hline $\mathrm{b}$ & 1322 & 860 & 466 & 30 \\
$\mathrm{c}$ & 1472 & 1090 & 470 & 44 \\
\hline $\mathrm{d}$ & 1566 & 1030 & 402 & 48 \\
\hline
\end{tabular}

For any survey data $\mathrm{x}$,define $\mathrm{h}_{T}(\operatorname{MUCH}(x))$ be the truth value function of fuzzy predicate $M U C H$, such that

$$
\mathrm{h}_{T}(\operatorname{MUCH}(x))= \begin{cases}0 & x<=\alpha_{F}+\varepsilon_{F} \\ \frac{d\left(x, \alpha_{F}+\varepsilon_{F}\right)}{d\left(\alpha_{F}+\varepsilon_{F}, \alpha_{T}-\varepsilon_{T}\right)} & \alpha_{F}+\varepsilon_{F}<x<\alpha_{T}-\varepsilon_{T} \\ 1 & x>=\alpha_{T}-\varepsilon_{T}\end{cases}
$$

$\alpha_{T}$ in the equation is the most accurate survey date of fuzzy predicate $\mathrm{MUCH}$ in tab2, $\varepsilon_{T}$ for elastic range of the data, $\alpha_{F}$ as the most accurate survey data of fuzzy predicate $\neg \mathrm{MUCH}, \mathcal{E}_{F}$ as its elastic range.

For high power generation, data in region $\mathrm{d}$ is the highest, so $\alpha_{T}=1566$, we can regard it as the most true for “ $M U C H$ ”,its elastic amplitude $\varepsilon_{T}=70$. For low power generation, data in region a is the lowest, so $\alpha_{F}=758$, we can regard it as the most truth for " $\neg M U C H$ ", its elastic amplitude $\varepsilon_{F}=20$. So, in terms of generating capacity:

$$
\mathrm{h}_{T}(\operatorname{MUCH}(x))= \begin{cases}0 & x<=778 \\ \frac{d(x, 778)}{d(778,1494)} & 778<x<1496 \\ 1 & x>=1496\end{cases}
$$

Thus, for any survey data, we can get the degree of the true value of predicate expression with corresponding truth value function.

\section{Establishment and significance of $\lambda$ in median logic infinite value model}

Take region c for an example to carry on the analysis, the high power generation in region $\mathrm{c}$ is 1472 , low power generation is 1090. According to the corresponding truth value function, we can get(rounded to three decimal places):

$$
\begin{aligned}
& h_{T}(\operatorname{MUCH}(1472))=\frac{d(1472-778)}{d(1496-778)}=0.966 \\
& h_{T}(\operatorname{MUCH}(1090))=\frac{d(1090-778)}{d(1496-778)}=0.435
\end{aligned}
$$

1472 and 1090 are a set of data reciprocally opposite. Theoretically $h_{T}(M U C H(1472))+h_{T}(M U C H(1090))=1$. 
However, due to fewer survey data, the data itself is not accurate enough,there are some differences between actual and model in the region, inevitably cause some error.

So let $\lambda=(0.966+0.565) / 2=0.766$, according to definition $2, \lambda$ represents the truth degree about predicate “ $\mathrm{MUCH}$ ” in region c., for any data $x$,if $h_{T}(\operatorname{MUCH}(x))>\lambda=0.766$, then $x$ is high power generation in region c,otherwise $x$ is low power generation.

So we get the meaning of $\lambda$, is numeric the regions.For example, if a specific survey data $\mathrm{x}$ if from region $\mathrm{c}$, then we use the parameter $\lambda$ corresponding to region $\mathrm{c}$, and if the data is from $b$, then we use the parameter $\lambda$ corresponding to region $b$. See table 3 .

Table 3 value of power generation $\lambda$

\begin{tabular}{|l|l|l|l|l|}
\hline $\begin{array}{l}\text { Power } \\
\text { generation }\end{array}$ & a & b & c & d \\
\hline$\lambda L$ & 0.837 & 0.863 & 0.766 & 0.875 \\
\hline
\end{tabular}

For power balance of this area, by applying the same method we can get the corresponding value of $\lambda$, as is shown in table 4:

Table 4 value of power balance $\lambda$

\begin{tabular}{|c|c|c|c|c|}
\hline $\begin{array}{l}\text { Power } \\
\text { balance }\end{array}$ & $\mathrm{a}$ & b & c & d \\
\hline$\lambda$ & 0.801 & 0.82 & 0.783 & 0.892 \\
\hline
\end{tabular}

\section{Fuzzy production rules and fuzzy reasoning}

After determine the value of $\lambda$ and the membership degree of corresponding fuzzy set of data $\mathrm{X}$, you can make appropriate decisions in accordance with the the grading rules prerequisite and match the extent of the data $\mathrm{X}$ 。To facilitate the process, we consider using the fuzzy production rules.

The general form of a fuzzy production rule is :

$Q \leftarrow P, C F, \tau$ or IF P THEN $Q,(C F, \tau)$

$\mathrm{P}$ represents a group of the premise or condition, $\mathrm{Q}$ represents certain conclusions or actions,Premise $\mathrm{P}$ and conclusions $\mathrm{Q}$ can be blurred. $\mathrm{CF}(0 \leq C F \leq 1)$ is called degree of confidence of the rules, $\tau(0 \leq \tau \leq 1)$ is a threshold。The meaning of this rule is "If the premise $\mathrm{P}$ in some degree be met by a certain degree of true can launch conclusion Q (or executive action Q), then the credibility of the rules is CF".

If premise of the rule is $\mathrm{MUCH}(\mathrm{t})$ and $\mathrm{x}$ belongs to region c. In table $3, \lambda=0.766$,if and only if $\operatorname{MUCH}(\mathrm{t}(\mathrm{x}))>0.766$,can explain $\mathrm{X}$ in $\mathrm{c}$ area belongs to high power generation.Accordingly, the characteristics of the parameter having certain similarities in some degree with the threshold value. When the rules confidence more than $\mathrm{x}$, this paper will take $\mathrm{x}$ value as the rule logic formula threshold,scilicet $\tau=\lambda$.

If the premise condition of rules is multiple,such as $Q \leftarrow P_{1}, P_{2}, \ldots, P_{n}, C F, \tau$, In this case $\tau$ Related to the collection $\left\{\lambda_{1}, \lambda_{2}, \ldots, \lambda_{n}\right\}$, then, $\tau=\max \left\{\lambda_{1}, \lambda_{2}, \ldots, \lambda_{n}\right\}$, where $\lambda_{i}$ is the parameter of the object which premise $P_{i}$ discussed in the region.

When the data $\mathrm{X}$ can satisfy a rule condition, execute the action part of the rule $\mathrm{Q}$, reasoning end.

Such as rule $1: \neg \operatorname{MUCH}(t(X)) \rightarrow \mathrm{A}$, the truth degree of class A depends on $\neg M U C H(t(X))$. Take this logical reasoning formula as production rule, The confidence of the rules can be obtained through a random survey, we suppose this credibility $\mathrm{CF}=0.96>\lambda$ 。 If the investigation of the object is in region A,then threshold $\tau$ can be the value of $\lambda=0.837$. Therefore, this rule can be expressed as: $A \leftarrow \neg M U C H(\mathrm{t}(X)), 0.96,0.837$.

Similarly, the rest of the rules can also be transformed into fuzzy production rules based on the survey object. For a specific data, Loop to find in the rule base. When X data can satisfy the condition part of a rule, then perform the action part of the rule.

\section{CONCLUSION}

Based on medium logic theory, the paper give a fuzzy knowledge representation and reasoning method, and take a smart grid transmission and distribution decisions for instance. This paper also give a method to determine the parameter $\lambda$ in medium predicate logic MF and the threshold $\tau$ in fuzzy production rules and automated reasoning algorithm based on this. These show that medium fuzzy logic system in the smart grid knowledge representation and reasoning is valid.

\section{REFERENCES}

[1] Zhu Wu-Jia,Xiao Xi-ArL Propositional Calculus System of Medium Logic(I)[J]. Nature Magazine,1985(8) : 315-316.

[2] Zadeh L Fuzzy sets[J]. Information and Control,1965(8) ; 338-353.

[3] Wang Dewen, Song Yaqi, Zhu Yongli, information platform of smart grid based on cloud computing, power system antomation.2010,34(22):7-12.

[4] Zhang Lizhen, Pan Zhenghua, fuzzy reasoning algorithm based on Intermediary logic, computer engineering and science,.2010,32(9):6568.

[5] Pan Zhenghua, et al. a infinite value semantic model and its significance, computer research and development,,2008,45(S1) : 158 -164 .

[6] Wang Cen, Pan Zhenghua, fuzzy knowledge representation and application based on medim logic, computer engineering and science.2008,30(11):80-82

[7] Xu Jiang, Pan Zhenghua, fuzzy knowledge and its different negative representation and reasoning in financial investment decision, computer application software.2011,28(3):37-40.

[8] Hong Long, Xiao Xi'an, Zhu Wugeng, Intermediary true value measurement and its application (I)[J], Chinese journal of computers, 2006,29(12) : 2186-2193.

[9] national electric power industry statistics bulletin. 